\title{
HIGH THROUGHPUT SOFT X-RAY SPECTROSCOPY WITH REFLECTION GRATINGS
}

\author{
Steven M. Kahn ${ }^{1}$ \\ ${ }^{1}$ Department of Physics and Space Sciences Laboratory, University of California, Berkeley, CA 94720 \\ USA
}

\begin{abstract}
As dispersing elements, grazing incidence reflection gratings offer the unique combination of high dispersion and wide spectral coverage at high efficiency. They can therefore be coupled with large area, low-resolution mirrors and high quantum efficiency detectors to yield moderate resolution spectroscopy of faint X-ray sources. Various design options are presented and compared, including both objective and convergent-beam configurations and both in-plane and off-plane grating mountings. A specific reflection grating payload design for ESA's X-Ray Multi-Mirror Mission (XMM) is reviewed in more detail. Predicted performance curves derived from ray trace studies are presented along with preliminary $\mathrm{X}$-ray reflectivity measurements of prototype grating samples.
\end{abstract}

\section{INTRODUCTION.}

In many astrophysical discussions of the scientific potential of $\mathrm{X}$-ray spectroscopy, primary emphasis has been given to the $\mathrm{Fe} \mathrm{K}$-line region between 6 and $7 \mathrm{keV}$. The $\mathrm{Fe} \mathrm{K}$ lines have been detected in a wide variety of sources, and have certainly provided useful physical constraints for many cosmic systems. However, the softer regions of the spectrum, particularly near $1 \mathrm{keV}$, may in the long run prove even more interesting. The soft $\mathrm{X}$-ray band $(0.2-2 \mathrm{keV})$ is densely permeated with important features, including the $\mathrm{K}$-shell transitions of $\mathrm{C}, \mathrm{N}$, and $\mathrm{O}$, and the L-shell transitions of $\mathrm{Fe}$. The $\mathrm{Fe}$ $\mathrm{L}$-shell lines are especially promising. They cover a very wide range of ionization and are quite wellseparated.

Unfortunately, it is the high density of expected transitions that makes it difficult to work in this band. Rather high spectral resolving power, $E / \Delta E \geq 200$, is required even to correctly identify the elemental species associated with observed features. Non-dispersive detectors, including the state-of-theart solid state devices, are not up to the task. Their resolution, $(\Delta \mathrm{E})$, is either fixed or increases slowly with energy, so that $(E / \Delta E)$ decreases at lower energies. Even the new cryogenic detectors (Holt 1989) are not sufficient below $1 \mathrm{keV}$ unless their resolution can be improved by at least a factor of ten. If spectral features cannot be uniquely identified in the raw data, then the power of high resolution spectroscopy to provide model-independent constraints becomes severely reduced.

For dispersive devices, on the other hand, the resolution in wavelength, $(\Delta \lambda)$, is approximately fixed across the band. Hence, for these cases, $(\mathrm{E} / \Delta \mathrm{E})$ increases at lower energies. The transmission grating spectrometers which were flown on Einstein and EXOSAT (Seward et al. 1982; Brinkman et al. 1980) exhibited this property; they achieved their highest resolving power at the lowest accessible energies. Transmission grating experiments are also planned for the upcoming SPEKTROSAT (Predehl $e t$ al. 1988) and AXAF (Schattenberg et al. 1988) missions.

In a dispersive spectrometer, an imaging optic must be incorporated somewhere in the system to provide the concentration. The limiting achievable spectral resolution scales essentially linearly with the angular resolution of the focussing mirror. A high resolution mirror is thus required to obtain the desired resolving power. Both AXAF and SPEKTROSAT will be $\sim$ arc-second facilities. 
The next really substantial increase in sensitivity for soft $\mathrm{X}$-ray spectroscopy, however, is more likely to be associated with the so-called "high throughput" missions like SPECTRUM X, XMM, and LAMAR (Peacock and Ellwood 1988; Gorenstein 1978). These experiments utilize an array of telescopes in order to obtain very large collecting area. In order to reduce costs, significant compromise in angular resolution is required. The design specifications for these facilities are more on the order of 0.5 to several arc-minutes.

With reduced angular resolution, very high dispersion is required to maintain the spectral resolution. It turns out that this is not possible with transmission gratings. The necessary groove densities are far in excess of current fabrication limits. Reflection gratings, on the other hand, do not suffer from this problem. A reflection grating used at grazing incidence can offer much higher dispersion than a transmission grating for a given line spacing. As is shown below, reflection gratings also yield fairly high diffraction efficiency in the soft X-ray band. Hence, when coupled to a high throughput mirror system, they provide a very sensitive, high resolution, soft $\mathrm{X}$-ray spectrometer.

In this paper, we review several important design considerations relevant to the incorporation of reflection gratings on high throughput $\mathrm{X}$-ray spectroscopy missions. We begin with the basic optics of reflection gratings in Section 2, and then move on to discuss grating orientation and various optical design options in Sections 3 and 4 respectively. We concentrate primarily on the motivations which have led to a specific design we are proposing for the XMM mission in collaboration with the Laboratory for Space Research at Utrecht. The optimization of this design and a brief summary of its expected performance and scientific capabilities is provided in Section 5. Finally in Section 6, we discuss grating fabrication issues and present some experimental results obtained for prototype grating samples.

\section{THE BASIC OPTICS OF REFLECTION GRATINGS.}

A schematic diagram of a simple reflection grating is given in Figure 1. We have defined a coordinate system in which the $\mathrm{Z}$-axis is parallel to the grating grooves and the $\mathrm{X}$-axis is oriented along the normal to the grating plane. An arbitrary incident ray makes an angle $\theta$ with the $Z$-axis and its projection in the XY-plane makes an azimuthal angle $\alpha$ with the $\mathrm{Y}$-axis. If the grating is large compared to all relevant scales, then the outgoing ray reflected off the grating will also have polar angle $\theta$. This is the so-called "conical diffraction" condition, since all outgoing rays emerge in a cone. The outgoing azimuthal angle, $\beta$, is related to the incoming angle, $\alpha$, by the dispersion equation:

$$
m \lambda=d \sin \theta(\cos \beta-\cos \alpha) \text {. }
$$

where $d$ is the groove spacing, and $m$ is an integer, i.e. the spectral order. Note that the zero order, $\alpha=$ $\beta$, corresponds to a pure reflection off the grating surface. The $m<0$ cases are referred to as the "inside orders" since the outgoing ray falls between the zero order and the normal on the cone. The $m$ $>0$ cases are referred to as the "outside orders".

Most applications which require high diffraction efficiency from the grating invoke "blazed" gratings in which the facets exhibit the staircase or sawtooth pattern illustrated in Figure 2 . The tilt angle of the facets, indicated by $\delta$ in the Figure, is called the blaze angle. Referring to the geometry above, we can calculate the incoming and outgoing graze angles made with the facet surface in terms of previous quantities:

$$
\begin{aligned}
\sin \gamma_{\text {in }} & =\sin \theta \sin (\alpha+\delta) \equiv \sin \theta \sin \mu_{\text {in }} . \\
\sin \gamma_{\text {out }} & =\sin \theta \sin (\beta-\delta) \equiv \sin \theta \sin \mu_{\text {out }} .
\end{aligned}
$$


These definitions apply to the inside order in particular. When $\gamma_{\text {in }}$ is equal to $\gamma_{\text {out }}$, then each of the facets behaves like a tiny mirror, and they all add coherently to give maximum diffraction efficiency. This is the "blaze condition". A simple manipulation of the dispersion equation shows that at blaze:

$$
\text { I } m \mid \lambda=2 d \sin \gamma \sin \delta .
$$

The wavelength which satisfies this condition for a given spectral order and for a given graze angle is called the blaze wavelength. Note that this expression holds for all polar angles, $\theta$.

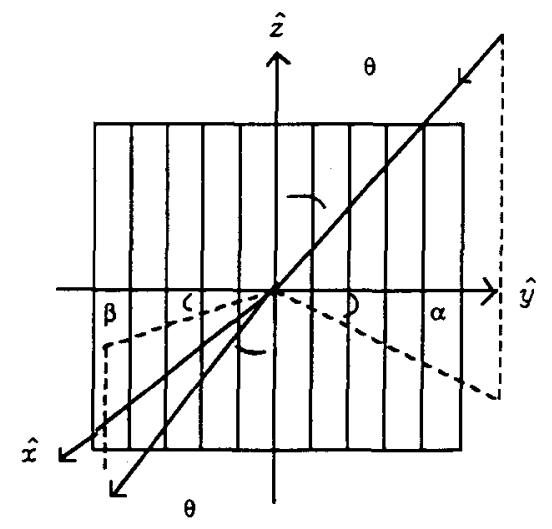
Figure 1: A schematic of a reflection grating showing the orientation of arbitrary incoming and outgo-
ing rays.

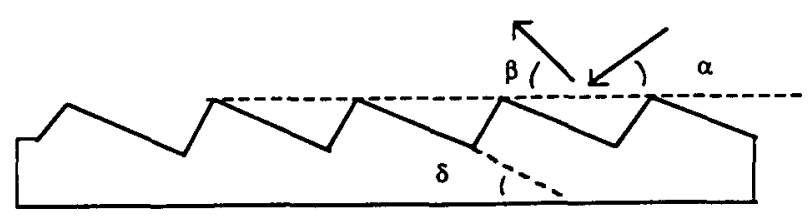

Figure 2: A blow-up of a blazed reflection grating illustrating the triangular shape of the grooves. The blaze angle $\delta$ is defined in this figure. 
For an ideal blazed grating, it is possible to apply Fraunhofer scalar diffraction theory to derive the diffraction efficiency of the grating in a given orientation for each of the spectral orders (Madden and Strong 1958). A straightforward calculation gives:

$$
E f f_{m}=\frac{g^{2}}{4 \sin ^{2} \theta \sin \alpha \sin \beta_{m}} P_{m}^{2}\left[\sin Q_{m} / Q_{m}\right]^{2}
$$

where:

$$
\begin{gathered}
g \equiv \sin \alpha / \sin (\alpha+\delta)=\frac{\sin \alpha}{\sin \mu_{i n}} . \\
P_{m} \equiv \sin \theta\left[\sin (\alpha+\delta)+\sin \left(\beta_{m}-\delta\right)\right] . \\
Q_{m} \equiv(\pi g d / \lambda) \sin \theta\left[\cos (\alpha+\delta)-\cos \left(\beta_{m}-\delta\right)\right] .
\end{gathered}
$$

Here $\beta_{m}$ is the outgoing angle for the mth spectral order. These expressions assume that the grating surface is perfectly reflecting. Of course, in the X-ray band, no surface is even close to perfectly reflecting and one must normalize by a reflection efficiency evaluated at some "effective graze angle". There is some debate in the literature over what is the correct normalization to use (Hutley 1982). The one we prefer involves the geometric mean of the reflectivities evaluated at the incoming and outgoing angles:

$$
\left[R\left(\gamma_{\text {in }}\right) R\left(\gamma_{\text {out }}\right)\right]^{1 / 2},
$$

which at least preserves the time reversal symmetry of the final expression.

Not surprisingly, even with the normalization above, the scalar diffraction calculations are only approximate. Because the incoming and outgoing graze angles are different, the reflection and diffraction aspects of the problem simply cannot be separated. The only correct approach is to selfconsistently solve Maxwell's Equations in free space subject to the material boundary conditions imposed at the grating surface. This is the "exact electromagnetic calculation" (Petit 1980). Comparisons show the largest discrepancy between the two calculations for the in-plane configuration, $\theta=90^{\circ}$, because the difference between $\gamma_{\text {in }}$ and $\gamma_{\text {out }}$ is largest in that orientation. Despite its inaccuracy, the simple diffraction theory does give the right qualitative behavior, and can be very useful for analytical optimization studies. The exact electromagnetic calculation is, of course, required for eventual instrument calibrations.

\section{GRATING ORIENTATION.}

In this Section, we address the question of the grating orientation, i.e. how should the grating be mounted with respect to the incoming beam? This issue has received a lot of attention in the literature in recent years, which has led to some confusion in the field. In order to compare the options fairly, it is necessary to parametrize the problem in an appropriate way and evaluate competing geometries which are individually optimized for the problem at hand. For a given wavelength band of interest, one must first select the first order blaze wavelength, $\lambda_{B}$, which can crudely be viewed as the wavelength of maximum sensitivity. The wavelength band also determines the graze angle, $\gamma$, which provides reasonable reflection efficiency. Hence, $\lambda_{B}$ and $\gamma$ can be regarded as fixed by scientific considerations. The other parameters are then coupled by the various relations. Specifically, $d$ and $\delta$ are related by the blaze equation (Equation 3) and $\theta$ and $\mu$ are coupled by the graze angle definition (Equation 2). Hence, in addition to $\lambda_{\mathrm{B}}$ and $\gamma$, there are at most two additional parameters which need to be specified. For reasons that will be clear later, our preferences for the parametrization are: $\mu$ and $\delta / \mu$. Since $\mu \equiv \alpha+\delta$ (for the inside orders) and $\alpha \geq 0, \delta / \mu$ must be $\leq 1$. 
There are, in fact, only two configurations which have been discussed extensively in the literature: the "in-plane" or "classical" configuration, in which the rays come in perpendicular to the grooves; and the "off-plane" or "conical" configuration, in which the rays come in nearly parallel to the grooves. In our notation, the in-plane case is characterized by $\theta=90^{\circ}, \mu=\gamma$, and the off-plane case by $\theta=\gamma$ and $\mu$ $=90^{\circ}$. There is a nice symmetry between them.

How does one choose between these two cases, or between any intermediary cases? Initially, we must look at the relative performance, i.e. the sensitivity and the resolution. This is easy to do analytically at the blaze wavelength itself. For example, the complicated Fraunhofer expression we had earlier (Equation 4) reduces to the simple form:

$$
E f f_{B}=R(\gamma) \frac{\sin (\mu-\delta)}{\sin (\mu+\delta)}
$$

when evaluated at the blaze. If the resolution is dominated by the blur introduced by the mirrors, $\varepsilon$, (as is true for most of the high throughput missions), and if this blur is essentially isotropic as seen by the grating, then another straightforward calculation gives the resolving power at blaze:

$$
(\lambda \Delta \lambda)=\frac{\sin \gamma}{\varepsilon}\left[\sin ^{2} \mu-\sin ^{2} \gamma+\frac{\sin ^{2}(\mu-\delta)}{4 \sin ^{2} \delta}\right]^{-1 / 2}
$$

For simplicity, we shall refer to the ratio of sines which appears on the right hand side of Equation 5 as the "sensitivity factor", $\eta$, and the term involving square brackets on the right hand side of Equation 6 as the "resolution factor", $\rho$. Clearly the optimal design will have the highest possible values of $\eta$ and $\rho$. In Tables 1 and 2 respectively, we list $\eta$ and $\rho$ as a function of the parameters we chose earlier. $\mu$ and $\delta / \mu$. These values are calculated for a prototype soft X-ray design: $\lambda_{B}=15 \AA$ and $\gamma=2^{\circ}$.

\begin{tabular}{|c||c|c|c|c|c|c|}
\hline \multicolumn{6}{|c|}{ Table 1-Sensitivity Factor $\eta$ Versus $\mu$ and $\delta / \mu$} \\
Calculated for $\lambda_{\mathrm{B}}=15$ Angstroms and $\gamma=2^{\circ}$ \\
\hline \hline$\delta / \mu$ । & $x$ & 50 & 10 & 20 & 60 & 90 \\
\hline 0.1 & 0.82 & 0.82 & 0.82 & 0.82 & 0.89 & 1.0 \\
\hline 0.3 & 0.54 & 0.54 & 0.34 & 0.53 & 0.68 & 1.0 \\
\hline 0.7 & 0.18 & 0.18 & 0.18 & 0.19 & 0.32 & 1.0 \\
\hline 0.9 & 0.053 & 0.053 & 0.054 & 0.057 & 0.11 & 1.0 \\
\hline 0.95 & 0.026 & 0.026 & 0.026 & 0.028 & 0.059 & 1.0 \\
\hline 1.0 & 0 & 0 & 0 & 0 & 0 & 1.0 \\
\hline
\end{tabular}




\begin{tabular}{|c||c|c|c|c|c|c|}
\hline \multicolumn{6}{|c|}{ Table 2 - Resolution Factor $\rho$ Versus $\mu$ and $\delta / \mu$} \\
Calculated for $\lambda_{B}=15$ Angstroms and $\gamma=2^{\circ}$ \\
\hline \hline$\delta \mu \mu$ & 20 & $5^{\circ}$ & $10^{\circ}$ & $20^{\circ}$ & $60^{\circ}$ & $90^{\circ}$ \\
\hline 0.1 & 0.22 & 0.22 & 0.22 & 0.23 & 0.25 & 0.30 \\
\hline 0.3 & 0.86 & 0.86 & 0.85 & 0.83 & 0.72 & 0.71 \\
\hline 0.7 & 4.7 & 4.4 & 3.7 & 2.5 & 1.2 & 0.97 \\
\hline 0.9 & 18 & 10 & 5.6 & 2.9 & 1.2 & 1.0 \\
\hline 0.95 & 38 & 12 & 5.8 & 2.9 & 1.2 & 1.0 \\
\hline 1.0 & $\infty$ & 13 & 5.9 & 2.9 & 1.2 & 1.0 \\
\hline
\end{tabular}

There are two conclusions which can be drawn from these tables: (1) First, there is an obvious complementarity between resolution and sensitivity. Parameter combinations that give high resolution give low sensitivity and visa versa. (2) Second, there is not actually that much variation in either parameter; for mid-range values of $\delta / \mu$, all values of $\mu$ give acceptable resolution and sensitivity. The in-plane design (left-most column) gives the highest resolution, whereas the off-plane design (right-most column) gives the highest sensitivity, but not by more than a factor of a few in either case. Interestingly, if we define a "figure of merit" as the product $\eta \times \rho$, it peaks at the two extreme cases, in-plane and off-plane, so there is, in fact, no real reason to consider the intermediate designs.

Evidently, there is not a strong case to be made for in-plane versus off-plane geometries based on theoretical performance alone. However, the two alternatives are distinguished in terms of practical considerations. In the high throughput soft $\mathrm{X}$-ray application, each requires pushing the fabrication state-of-the-art in a particular direction. For the in-plane mount, this involves fabricating low blaze angles. Since $\mu=\gamma=2^{\circ}$ in this case, and since $\delta$ is a fraction of $\mu, \delta$ is typically required to be less than $\sim 1^{\circ}$. $1.5^{\circ}$ blaze angles are easily fabricated, but $<1^{\circ}$ is conventionally viewed as a technical challenge. For the off-plane mount, the problem involves very high groove density. Since $\mu=90^{\circ}$ in this case, $\delta \equiv$ tens of degrees, and $d$ must be much smaller. In fact, virtually all of the off-plane designs shown in the tables require groove densities $>40,000 \mathrm{~V} / \mathrm{mm}$. That is far beyond the state-of-the-art for reflection gratings by at least a factor four. As it turns out, the low blaze angles required for the inplane designs are much less of a concem (see Section 6), so, by this analysis, the in-plane configuration appears to be preferred for the high throughput X-ray application.

\section{OPTICAL DESIGN.}

We now turn to questions of the optical design. In particular, where should the grating be placed with respect to the $\mathrm{X}$-ray optical path? At longer wavelengths, a reflection grating is typically located behind a slit in the telescope focal plane, as in the classical Rowland circle design. However, since the grating must be used at grazing incidence in the soft $\mathrm{X}$-ray band, this configuration introduces a number of technical problems, the most important of which is that it requires an extremely long $(-2 \mathrm{~m})$ instrument bay behind the focal plane. There are some tricks which can be invoked to partially get around this difficulty, but not enough so to make it easy to incorporate this kind of design.

Another possibility is to mount the grating in front of the telescope itself (Cash 1988). This has the advantage that it does not disturb the telescope focal plane assembly. The grating is tilted and the 
telescope is offset pointed from the source so that the desired wavelength band is diffracted down the optical axis. In principle, one can convert between direct imaging and spectroscopic modes by simply rotating the grating in and out of the field of view of the telescope.

The principal disadvantage of this configuration is that the telescope is used as a camera to image the spectrum, i.e. one relies on both its on-axis and off-axis properties. Conventional X-ray telescopes have, by optical standards, very poor off-axis performance; aberrations grow quadratically with off-axis angle. In addition, vignetting by the mirror shells seriously limits throughput off-axis. Hence, the spectrum is degraded near the edges of the band.

As an alternative, the grating can be placed behind the mirror. The telescope is then used on-axis at all wavelengths. However, in this configuration, the grating does not see parallel light. That introduces aberrations if not properly corrected. As first shown by Hettrick and Bowyer (1983), the aberrations can be removed if the groove spacing is varied across the grating plane. Hettrick's varied linespace solution has the additional advantage that it yields a focal plane which lies at near normal incidence to the beam. A potential drawback to placing the grating behind the telescope is that the detector must then be offset from the telescope focal plane. Thus, this configuration is not easily convertible between imaging and spectroscopic applications.

Since the grating is used at exteme grazing incidence in the soft X-ray band, a very large grating would be required to completely cover the beam exiting a typical high throughput telescope. A single large grating can be assembled from a set of smaller gratings arrayed in a co-planar configuration, however this solution is prohibitively expensive. Since the required groove spacing depends on the distance from telescope focus, the individual smaller gratings cannot be identical. In addition, this approach leads to reduced angular resolution, since the angular blur introduced by the mirrors increases nearly linearly with distance down the optic axis of the telescope.

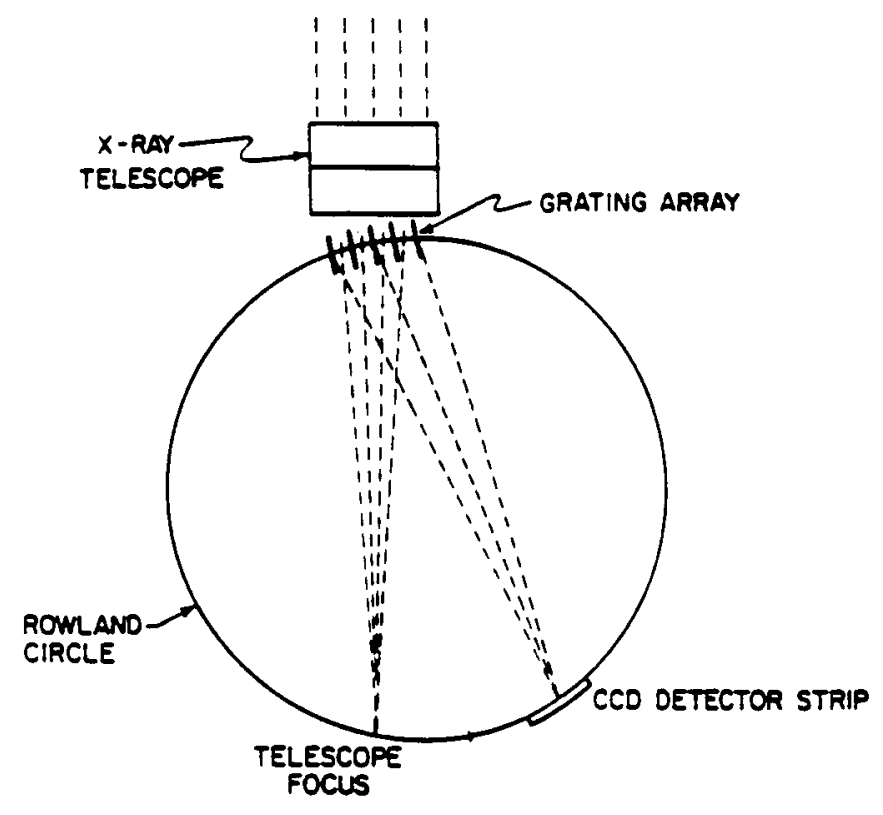

Figure 3: The reflection grating optical design we are proposing for incorporation on XMM. The gratings sit on a Rowland circle in a near-parallel stack at the exit from the $\mathrm{X}$-ray mirror. 
Alternatively, a set of gratings can be arrayed alongside each other in a near parallel stack (see Figure 3). In this case, the gratings can be identical replicas of a single master since they all lie at roughly the same distance from the telescope focus. A truly parallel array will generate aberrations. However, these can be cured by an appropriate choice of geometry. The array is arranged so that all gratings are oriented at the same angle with respect to the incident ray at grating center. In addition, the gratings are aligned along a Rowland circle, as shown in Figure 3, which also includes both the telescope and spectroscopic foci. If the spectroscopic detectors are aligned along this circle, comatic aberrations associated with the array are eliminated for all wavelengths in the spectrum.

A complication arises with this design due to vignetting of outgoing rays by neighboring gratings in the stack. Since the outgoing rays leave the grating at larger angles than the incident rays, this can only be corrected by spacing the gratings at larger separations, i.e. by not intercepting the entire beam exiting the telescope. The unintercepted rays can be effectively utilized by a complementary instrument in the telescope focal plane. This yields a kind of "built-in redundancy" in the use of the telescope, with no moving parts.

\section{OPTIMIZATION AND SCIENTIFIC PERFORMANCE OF THE XMM DESIGN.}

Motivated by the considerations outlined above, we are proposing a reflection grating spectrometer for XMM which consists of an array of thin reflection gratings placed in the Rowland circle configuration at the exit from the $\mathrm{X}$-ray telescope. The gratings are mounted in the in-plane configuration and are oriented so that the first, second, and third inside spectral orders are diffracted at moderate to high efficiency. The diffracted light is imaged by an array of charge-coupled device (CCD) detectors offset from the telescope focal plane. The separation of the spectral orders is accomplished using the energy resolution of the CCDs.

The complete specification of the optical design for this configuration requires a choice of grating parameters, specifically the average line spacing on the gratings, $d$, the mean incidence angle, $\alpha$, and the blaze angle, $\delta$. These in turn determine the length of the detector array and its position with respect to the telescope focus. The optimization begins with a choice of first order blaze wavelength. Given the prevalence of important spectral features between 0.5 and $1 \mathrm{keV}$, we choose $\lambda_{\mathrm{B}}=15 \AA$. $\mathrm{d}$, $\delta$, and the graze angle, $\gamma$ are then coupled by the blaze equation (Equation 3). Hence, only two of the three parameters are sufficient to specify the design. It is convenient to choose $\gamma$ and $\eta=\sin (\gamma-\delta) / \sin (\gamma+\delta)$ as the relevant parametrization. The resolution of the design clearly increases as we increase the detector length. Hence the number of $\mathrm{CCD}$ chips required becomes the limiting consideration. In terms of $\gamma$ and $\eta$, the detector length is approximately given by:

$$
l_{D}=\frac{2 \gamma L}{(1+\eta)}\left[\left(\eta^{2}\left(1-\frac{\lambda_{\max }}{\lambda_{B}}\right)+\frac{\lambda_{\max }}{\lambda_{B}}\right\}^{1 / 2}-\left\{\eta^{2}\left(1-\frac{\lambda_{\min }}{\lambda_{B}}\right)+\frac{\lambda_{\min }}{\lambda_{B}}\right\}^{1 / 2}\right],
$$

where $L$ is the distance between the center of the grating box and the telescope focal plane, and $\lambda_{\max }$ and $\lambda_{\min }$ are the maximum and minimum wavelengths in the spectral band respectively. For a $\max$ max imum allowable detector length, there is a coupling between $\eta$ and $\gamma$, with $\gamma$ an increasing function of $\eta$.

The total diffraction efficiency of the system at blaze also depends on these two parameters. From the scalar Frauhofer theory (which is a reasonable approximation near the blaze), we get:

$$
\text { Effic }=\eta^{2} R\left(\gamma, \lambda_{B}\right)
$$

where $R\left(\gamma, \lambda_{B}\right)$ is the reflection efficiency of the surface at angle $\gamma$ and wavelength $\lambda_{B}$. Since $\gamma$ increases with $\eta$ to maintain the detector length, and $R\left(\gamma, \lambda_{B}\right)$ is a sharply decreasing function of $\gamma$, the 
efficiency is maximized at a well-defined optimal set of $\eta, \gamma$ values.

For $\mathrm{XMM}$, we take $\lambda_{\max }=35 \AA, \lambda_{\min }=5 \AA$, and $\mathrm{L}=6.7 \mathrm{~m}$. We assume a maximum detector length of $235.8 \mathrm{~mm}$. We find the maxium mix of first and second order efficiencies at an optimal value of $\eta$ of 0.53 . The implied blaze angle is $0.70^{\circ}$ and the mean groove density is $646 \mathrm{1} / \mathrm{mm}$. The mean incident angle on the gratings is $1.58^{\circ}$.

The effective area for this optimized design incorporated behind two of the three XMM telescopes is illustrated in Figure 4. Separate curves are given for the first, second, and third spectral orders diffracted by the gratings. As can be seen, the effective area of the system is extremely high, greater than $100 \mathrm{~cm}^{2}$ over most of the instrument bandpass. The resolving power is shown as a function of wavelength in Figure 5 for first and second spectral orders. As can be seen, it exceeds the requirement $\lambda / \Delta \lambda>200$ over the entire band. This resolution is more than adequate to yield unique identifications for nearly all discrete features expected to be prominent in soft X-ray spectra of cosmic sources.
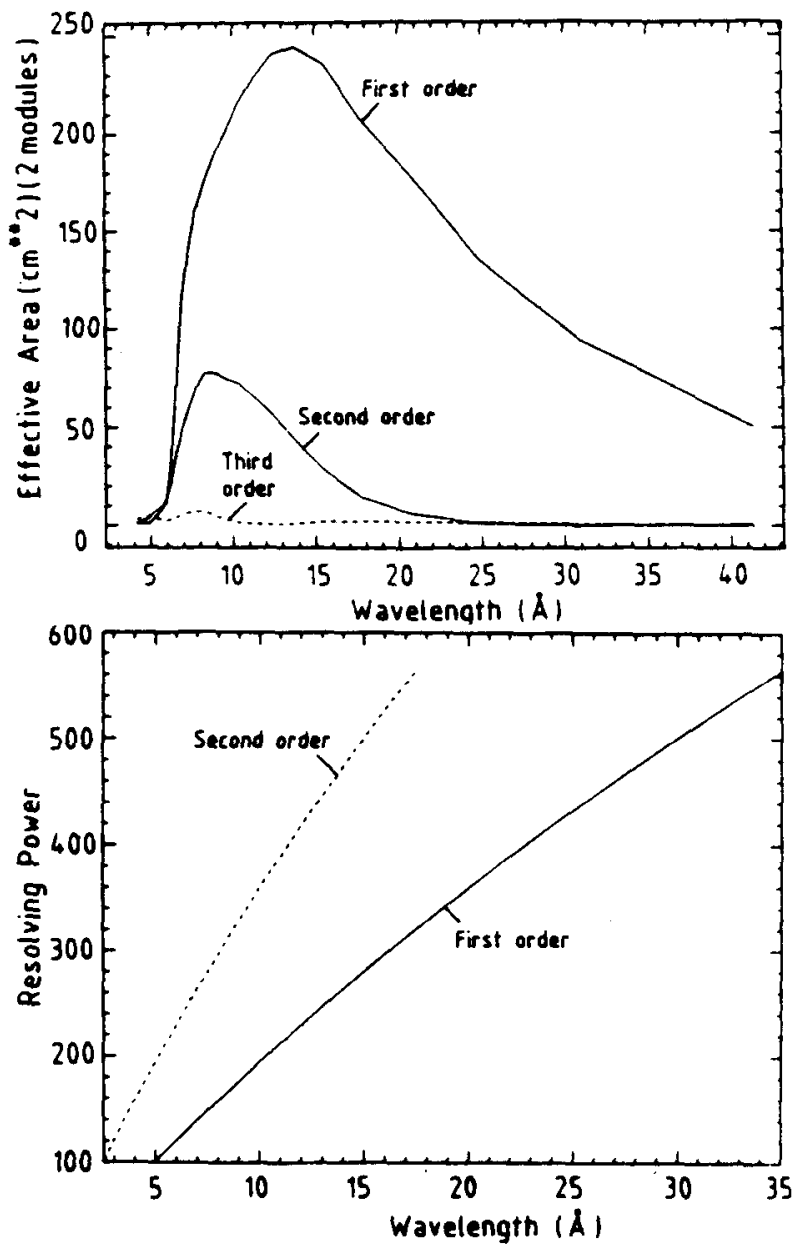

Figures 4 (top) and 5 (bottom): The effective area and resolving power respectively as a function of wavelength for the proposed XMM design. 


\section{FABRICATION ISSUES.}

We now turn to the question of fabrication, can the required reflection gratings be made with current technology? As mentioned earlier, the principal challenge for the in-plane design involves the low blaze angle, $\delta=0.70^{\circ}$. However, since the required line spacing is rather coarse, $1 / \mathrm{d}=646 \mathrm{l} / \mathrm{mm}$, the actual groove depth is not much less than has been previously fabricated.

There are two methods of producing gratings: by mechanical and holographic means (Michette 1986). Mechanical ruling is a burnishing process, a diamond stylus is dragged across the surface displacing the metal coating. At high pressures, the metal is fluid; the process is more akin to plowing a path through snow rather than cutting into metal. The groove shape is not well-defined by a single pass. It only begins to take its final shape when the neighboring grooves ae ruled as well. Hence, it is difficuit to control accurately. However, the varied spacing required of our design does not present a problem for this technique. Since each groove is independently ruled, its position can be precisely controlled.

The holographic technique uses a photoresist exposed to a laser interference pattern to create the periodic structure. The result is then developed and ion-etched in a controlled manner to produce the desired triangular groove profile. Since this is not a mechanical process, it yields more predictable results. However, there is some question as to whether the required continuous variation in groove spacing can be produced with this technique.

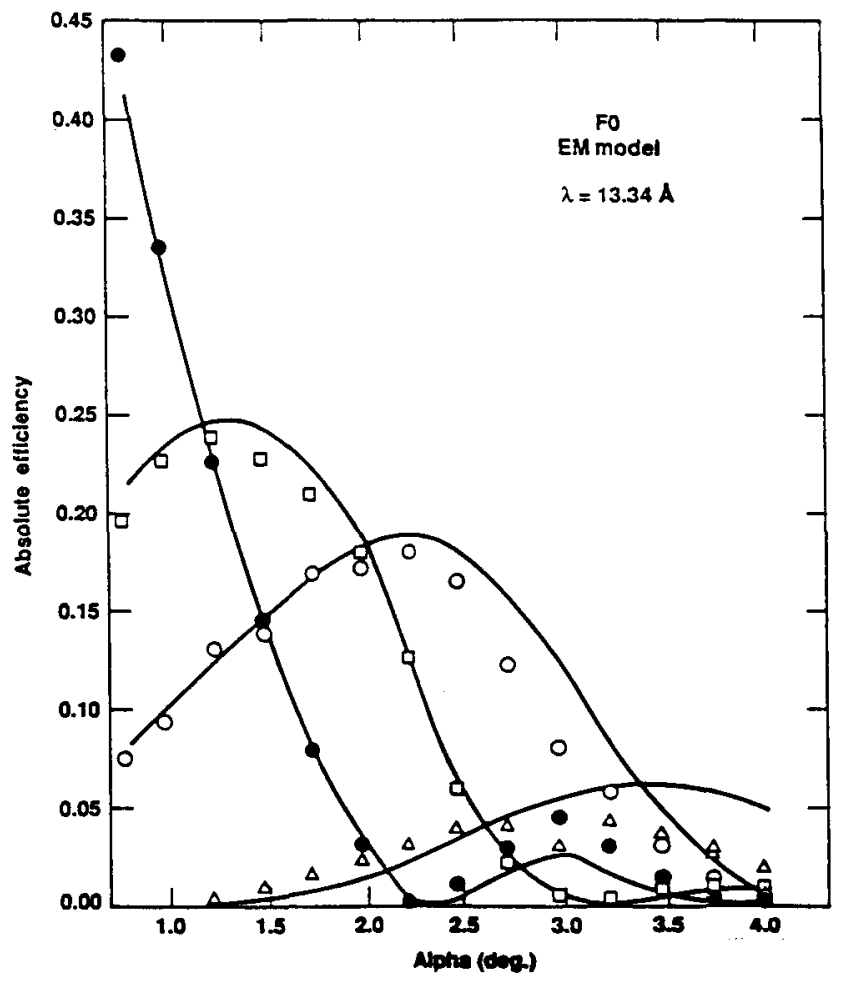

Figure 6: Measured absolute reflectivities in 0th (filled circles), 1st (open squares), 2nd (open circles), and 3 rd (open triangles) spectral orders at $13.34 \AA$, plotted as a function of incident angle for a holographic prototype XMM grating. The solid lines give the predicted reflectivities from the exact electromagnetic theory assuming a perfect blazed groove shape. (From den Boggende et al. 1988). 
In connection with the XMM proposal effort, we have acquired prototype grating samples of both varieties and tested them in dedicated facilities at both Utrecht and Livermore (den Boggende et al. 1988). The high diffraction efficiency expected has, in fact, been verified for both cases. The results for the holographic sample are shown in Figure 6. Here 0th, 1st, 2nd, and 3rd order absolute reflectivities at $13 \AA$ are plotted as a function of incident angle. The solid lines give the theoretical predictions using the exact electromagnetic treatment. As can be seen, the agreement with the data is excellent, indicating that the groove shape exhibits a nearly perfect blaze profile. Further tests with varied space samples are currently in progress.

\section{SUMMARY.}

Reflection gratings offer special promise for incorporation on upcoming high throughput $\mathrm{X}$-ray spectroscopy missions where limited angular resolution in the X-ray telescope prevents use of conventional transmission gratings. A variety of general considerations suggest that an optical design incorporating an array of gratings mounted in the in-plane configuration behind the $\mathrm{X}$-ray mirror is particularly attractive for this application. A reflection grating spectrometer of this design is currently being proposed for the XMM mission. Preliminary measurements of prototype grating samples have verified that it is possible to achieve near theoretical reflectivity at the appropriate soft $\mathrm{X}$-ray wavelengths.

\section{REFERENCES.}

Brinkman, A.C., Dijkstra, J.H., Geerlings, W.F.P.A.L., van Rooijen, F.A., Timmermann, C., and de Korte, P.A.J., 1980, Appl. Opt., 19, 1601.

Cash, W., 1988, Proc. SPIE, 830, 204.

den Boggende, A.J.F., de Korte, P.A.J., Videler, P.H., Brinkman, A.C., Kahn, S.M., Craig, W.W., Hailey, C.J., and Neviere, M., Proc. SPIE, 982, 283.

Gorenstein, P., 1978, in New Instrumentation for Space Astronomy, ed. K. van den Hucht and G.S. Vaiana, Oxford, Pergamon Press, p. 232.

Hettrick, M.C., and Bowyer, S., 1983, Appl. Opt., 22, 3921.

Holt, S.S., 1989, this volume.

Hutley, M.C., 1982, Diffraction Gratings, Academic Press, London.

Madden, R.P., and Strong, J., 1958, Diffraction Gratings, Appendix P, in Concepts in Classical Optics, Freeman and Co., San Francisco.

Michette, A.G., 1986, Optical Systems for Soft X-Rays, Chapter 7, Plenum Press, New York.

Peacock, A., and Ellwood, J., 1988, Proc. SPIE, 982, 277.

Petit, R., 1980, Electromagnetic Theory of Gratings, in Topics in Current Physics, 22, Springer Verlag, Berlin.

Predehl, P., Brauninger, H., Burkert, W., Aschenbach, B., Trumper, J., Kuhne, M., and Muller, P., 1988, Proc. SPIE, 982, 265.

Schattenburg, M.L., Canizares, C.R., Dewey, D., Levine, A.M., Markert, T.H., and Smith, H.I., 1988, Proc. SPIE, 982, 210.

Seward, F.D., Chlebowski, T., Delvaille, J.P., Henry, J.P., Kahn, S.M., Van Speybroeck, L., Dijkstra, J., Brinkman, A.C., Heise, J., Mewe, R., and Schrijver, J., 1982, Appl. Opt., 21, 2012. 